\title{
Method of Construction of Routes of the Unmanned Aerial Vehicle on the Interactive Electronic Map
}

\author{
Tatiana Mikheeva \\ Institute of aviation technology \\ Samara University \\ Samara, Russia \\ mikheevati@gmail.com \\ Nikita Ostroglazov \\ Institute of informatics, mathematics \\ and electronics \\ Samara University \\ Samara, Russia \\ nickitaost@gmail.com
}

\author{
Aleksey Tikhonov \\ Institute of aviation technology \\ Samara University \\ Samara, Russia \\ oupt_ssau@mail.ru \\ Sergey Mikheev \\ Institute of aviation technology \\ Samara University \\ Samara, Russia \\ ms140-500@yandex.ru
}

\author{
Elena Chekina \\ Institute of aviation technology \\ Samara University \\ Samara, Russia \\ ev-chekina@yandex.ru
}

\begin{abstract}
Unmanned aerial vehicles are increasingly used to perform complex transport processes and operations, such as monitoring transport processes, photogrammetry of transport infrastructure, inspection of geoobjects and tracking of mobile agents. The construction of rational routes in solving the problems of management of the functioning of the transport infrastructure, taking into account various criteria, is a complex procedure that requires reducing the time, improving the trajectory. The use of heuristic algorithms, for example, the ant colony algorithm, which has high accuracy and speed of solving problems, is relevant. To perform complex technological processes and operations, unmanned aerial vehicles (UAVs) are increasingly used that can replace a human operator in dangerous living conditions. To implement these processes, it is necessary to control the flight. As a result, it is revealed that the advantage of the ant algorithm method is the possibility of finding a rational route, which is achieved by repeated repetition of the procedure of passing agents along the route.
\end{abstract}

Keywords-unnamed aerial vehicle, route, intelligent transport geoinformation system, route, $U A V$

\section{INTRODUCTION}

To perform complex technological processes and operations, unmanned aerial vehicles (UAVs) are increasingly used that can replace a human operator in dangerous living conditions. To implement these processes, it is necessary to control the flight. In connection with the global trend of increasing the level of UAV autonomy in solving the set objectives, the role of using intelligent autopilots, implemented, for example, on the basis of intelligent transport systems, is increasing.

Determining the rational route of the UAV when performing tasks in different operating conditions is a complex and lengthy procedure associated with a variety of options for choosing from possible solutions.

\section{THE PROBLEM OF ROUTING UAVS}

The complexity of the problem is to determine a set of points in space that would meet the trajectory of the UAV and displayed on the map. The choice of route is influenced by factors:
- limited flight time;

- flight safety;

- multiple simultaneous routes.

Limiting flight time involves determining the route that would allow the UAV to have a sufficient battery level to perform the task and return to the charging station. For practical reasons, the UAV route should consist of straight sections and curvature sections connecting straight sections.

Flight safety is determined by a set of relevant restrictions: compliance with a given level and flight corridor, overflight of obstacles on the way, the distance to the emergency landing platform, the distance from the no-fly zones, changes in weather conditions. In the case of taking into account the first and second factors, there is a joint problem, both reducing the route time and reducing the length of the trajectory.

The last factor indicates the ambiguity of the way in which UAVs can follow. The solution of this problem requires the nomination of a criterion by which to choose the most suitable route $[1,2,3]$.

\section{A MATHEMATICAL MODEL OF THE TRANSPORT NETWORK}

The described problem of making rational UAV routes with their visualization on an interactive electronic map is mathematically formulated as follows.

The transport network of the city including the street and road network (UDS) which model is displayed on the electronic map in the environment of intellectual transport geoinformation system "ITSGIS" is set. ITSGIS allows you to use different types of visual geomodels: points, lines, polygons. We introduce the following notations.

If $\Theta=\theta_{j} X \quad(\Theta \neq \varnothing)-$ a transport network of any configuration that contains the following subsets, as shown in Fig. 1:

$\Theta^{Z} \subset \Theta-$ many areas are points of departure;

$\Theta^{D} \subset \Theta-$ many of the areas destinations; 
$\Theta^{A} \subset \Theta-\mathrm{a}$ lot of zones, charging stations;

$\Theta^{F} \subset \Theta$ - many areas - prohibited areas;

$\Theta^{H} \subset \Theta$ - many zones - railway crossings;

$\Theta^{P} \subset \Theta$ - many areas - pedestrian crossings;

$\Theta^{S} \subset \Theta-$ many areas - crossroads;

$\Theta^{L} \subset \Theta-$ many areas - hauls;

$\Theta^{U} \subset \Theta$ - multiple zone - tunnels;

$\Theta^{o} \subset \Theta-$ many areas of the overpasses.
Zone «A point of departure» $\Theta^{Z}=\theta_{i}^{Z}, i=1,2, \ldots, n$ it is a section of the transport network that determines the place (point on the map) of departure and loading of the UAV.

Zone «Charging station» $\Theta^{A}=\theta_{i}^{A}, i=1,2, \ldots, n$ it is a section of the transport network on which the UAV is recharged.

Zone «Forbidden territory» $\Theta^{F}=\theta_{i}^{F} \quad, i=1,2, \ldots, n$ it is a section of the transport network over which UAV flights are prohibited due to the peculiarities of the security mode of the object.

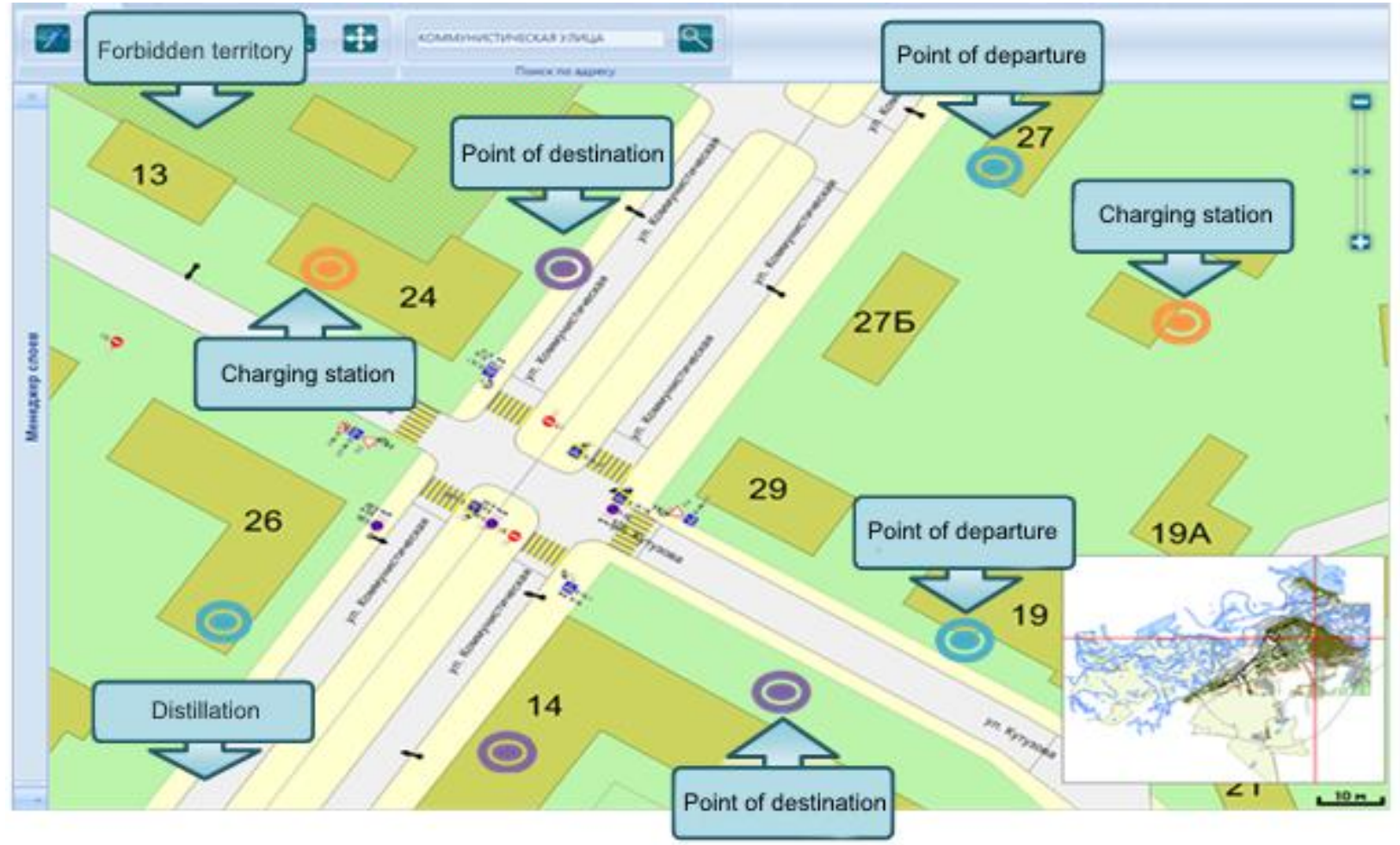

Fig 1. Transport network zones on the electronic map

Zone «Railway crossing» $\Theta^{H}=\theta_{i}^{H} \quad, i=1,2, \ldots, n-$ the section of the road network that crosses the railway, which is on the same level with it.

Zone «Pedestrian crossing» $\Theta^{P}=\theta_{i}^{P} \quad, i=1,2, \ldots, n-$ the section of the road network on which there is a land pedestrian crossing limited by road signs and/or borders of the intersection; has the form of a polygon (quadrangle). The width of a pedestrian crossing is determined by the distance between road signs or the width of the road markings.

Zone «Crossroads» $\Theta^{s}=\theta_{i}^{s}, i=1,2, \ldots, n$ - the section of the road network, located at the intersection of two or more roads, has its own unique geometric shape, completely repeating the shape of the real intersection.
Zone «Distillation» $\Theta^{L}=\theta_{1}^{L}, i=1,2, \ldots, n$ - the linear section of the street and road network, which is not a section of the type "intersection", "pedestrian crossing", "railway crossing"; located between sections of other types, including other crossings, has the form of a polygon (often a quadrangle).

Zone «Tunnel» $\Theta^{U}=\theta_{i}^{U} \quad, i=1,2, \ldots, n$ - the linear section of the street-road network, located between the sections of the "stage" type, has the shape of a polygon (often a quadrangle); it has limitations in size in height and width.

Zone «Overpass» $\Theta^{o}=\theta_{i}^{o}, i=1,2, \ldots, n$ - the linear section of the street and road network, located between the sections of the "stage" type, has the shape of a quadrangle; it has restrictions on the height and width of the dimensions, as 
well as specific flight conditions of the UAV, complicating movement, for example, the presence of a large number of power lines wires or supporting supports.

For a full description of the transport network, including the street and road network and the zone of dispatch, delivery, charging, taking into account the direction of movement of the UAV between them, objects are additionally introduced: the node and the arc, which are elements of the oriented graph $[13,14]$.

Geometric oriented graph $\mathrm{G}$ in space $\mathfrak{R}^{n}$ we will consider the set of a non-empty set $\tilde{V}=\mathfrak{\mathfrak { h }}_{\text {i }}$ objects of some nature in $\mathfrak{R}^{n}$, called vertices (nodes) of a graph, a set of $\widetilde{E}=\left\{\tilde{e}_{i}\right.$ continuous self-intersecting curves in $\mathfrak{R}^{n}$, called edges (arcs) of the graph, incidentor $\widetilde{P}_{1}$ assigns to each arc $\tilde{e}_{i} \in \tilde{E}$ top $\tilde{v}_{i} \in \tilde{V}$, called the beginning of an arc, an incidentor $\tilde{P}_{2}$, matching the arc $\tilde{e}_{i} \in \tilde{E}$ top $\tilde{w}_{i} \in \tilde{V}$, called the end of an arc satisfying the following conditions:

- $\quad$ each open curve in $\tilde{E}$ contains exactly two points of the set $\tilde{V}$, which are its boundary points;

- curves in $\tilde{E}$ do not have common points, except for points from the set $\tilde{V}$.

- $\tilde{E} \subset \tilde{V} \times \tilde{V}$.

In the mathematical model considered in the work, a specialized graph is used, the vertices of which at the intersection correspond to stop lines at the approaches to intersections, at a pedestrian crossing and a railway crossing are the boundaries of the site, at the stretch correspond to the boundaries of the site and the points of possible branching of flows, and the arcs are all possible paths of the UAV movement along the next to the current section of the UDS.

When designing a transport network based on objectoriented programming, the triad of objects <section>, <node>, <arc> (Fig. 2) is the basis of the conceptual model of ITSGIS, all other objects will be, one way or another, tied to this basis.

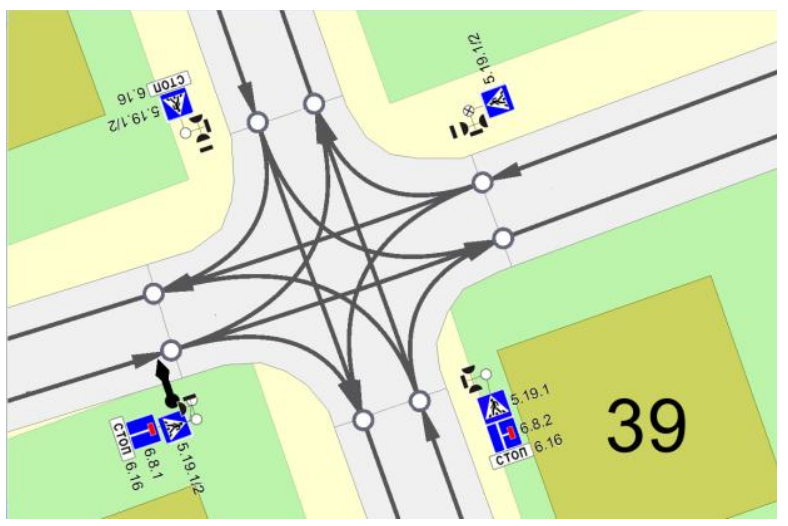

Fig. 2. Nodes and arcs of the road network on the interactive map
$<$ Plot $>$ - polygonal section of the transport network different zones, represented by a set of coordinate pairs, described by a single set of physical parameters. In the ITSGIS environment, a parcel is encoded by a polygon defined by the coordinates of its vertices.

<Host> is the location of the flow separation of the vehicles. The node is the top of the oriented graph, channeling traffic flows, always lies at the junction of two sections and shows the possibility of movement from one section to another in the direction determined by the corresponding arc. Two adjacent plots are matched by two nodes. In the environment ITSGIS node is encoded by the point [4].

$\langle A \boldsymbol{r} \boldsymbol{C}\rangle$ is an element of a directed graph that specifies the direction of traffic flows (or with the detail of vehicles) on the site and contains the relevant characteristics (arc length, traffic intensity in this direction, flow density, etc.). One arc defining the direction of movement of vehicles or two arcs defining the place of a turn (change of the direction of movement) is put in correspondence to two nodes. Multiple arcs (streams) can pass through a single parcel, but the arc can only be within a single parcel. In the environment ITSGIS arc is a linear object $[15,16]$.

On the transport network, the movement of UAV groups is carried out by the total number of $Q$ from the point of departure (charging station) $\Theta^{Z}\left(\Theta^{A}\right)$ to the destination $\Theta^{D}$, in order to perform some tasks (UDS survey, photo or video recording, delivery of small cargo).

If $\tilde{D}=\tilde{d}_{i}{ }^{X} \quad \tilde{D} \neq \varnothing \quad$ - many unmanned aerial vehicles, which contains the characteristics of different types of $X$ UAVs:

$\tilde{D}=\tilde{D} w_{d_{i}}, l_{d_{i}}, h_{d_{i}}, p_{d_{i}} \quad-$ many characteristics of the UAV $v=1,2, \ldots, q$, where $w_{d_{i}}-$ the width of the UAV $\tilde{d}_{i}^{X}$, $l_{d_{i}}$ - the length of the UAV $\tilde{d}_{i}^{X}, h_{d_{i}}$ - the altitude of the $\mathrm{UAV} \tilde{d}_{i}^{X}, p_{d_{i}}-$ the capacity of a UAV $\tilde{d}_{i}^{X}$;

$q_{i}$ - demand in the goods associated with each customer;

$Q_{P}$ - the amount of cargo required to pick up from the customer [5].

\section{ROUTE CONSTRUCTION METHOD}

The tasks of constructing rational UAV routes are key in the field of logistics and are NP-complex tasks of combinatorial optimization. In this regard, the most relevant is the development and application of heuristic algorithms. There are precise methods for determining optimal routes, but they require exponential computation time. Therefore, much attention is paid to the development of so-called metaheuristic algorithms, for example, the use of a multiagent approach with high accuracy and speed of solving problems [6]. Multi-agent systems, borrowed from public Biosystems, which include the anthill, are attractive on some grounds, such as autonomy of individual agents, decentralization of control through the implementation of collective intelligence in the system and trainability of agents 
[7]. The ant colony algorithms were first proposed by the authors Dorigo, Maniezzo, Solorni as a method for solving difficult combinatorial optimization problems such as the traveling salesman problem and the quadratic assignment problem [8]. When using this algorithm, the best route is determined by repeatedly exploring the search space in different directions. A feature of the proposed movement is the presence of pheromone released by agents on the way in the process of its movement. The best route is the one with the highest pheromone content. The ant colony algorithm is one of the meta-heuristics algorithms used to solve the traveling salesman problem $[9,10]$.

The ant colony algorithm has several modifications. One of the algorithms used in the construction of solutions from iteration to iteration only the best solutions obtained in previous iterations is the algorithm Ant Colony Optimization Based on Population (P-ACO) [5].

The initial step of the algorithm is to initialize the pheromone level. Then, at each iteration, the further direction of movement is selected from the current location with probability according to the formula:

$$
\rho c_{j} \mid s c_{k}=\left\{\begin{array}{l}
\frac{\tau_{j}^{\alpha} \cdot \eta_{j}^{\beta}}{\sum c_{i} \in L s c_{k} \tau_{j}^{\alpha} \cdot \eta_{j}^{\beta}}, \text { if } c_{i} \in L s c_{k} \\
0, \text { otherwise }
\end{array}\right.
$$

where $\alpha, \beta$-the parameters of the algorithm, showing the influence of the level of pheromone $\tau$ and heuristic information $\eta$ for each decision of the agents, respectively;
$L \begin{array}{llll} & c_{k}\end{array}$-directions that the agent $\alpha \in A$ not yet added to the partially built solution $s_{\alpha} c_{k}$ with the last component $c_{n}$. In the next iteration, the solutions obtained in the previous iteration are used to override the pheromone values according to the rule defined by the formula:

$$
\tau_{i}=1-\rho \times \tau_{i-1}+\sum_{\alpha \in A} \Delta \tau_{j}^{s_{\alpha}}\left(\tau_{i j}+1-\rho \times \tau_{i j}+\sum_{\alpha \in A} \Delta \tau_{i j}^{s_{\alpha}}\right)
$$

where

$\Delta \tau_{i j}^{s_{\alpha}}=\left\{\begin{array}{l}\frac{k}{l s_{\alpha}}, \text { if } c_{j} \text { used in the solution } s_{\alpha} \\ 0, \text { otherwise }\end{array}\right.$

$l \quad s_{\alpha}$ - the length of the path of the agent $\alpha$;

$\rho$ - coefficient, $<-\rho_{-}$represents the evaporation of pheromone, $0<\rho<1$;

$k$ - constant close to 1 .

As can be seen from the formula, the pheromone value in decision-making increases on the components of the best decisions and, accordingly, decreases on the components of the worst decisions. The criterion for closing the algorithm cycle can be a given number of iterations or the number of iterations that do not bring an improvement in the expected solution $[11,12]$.

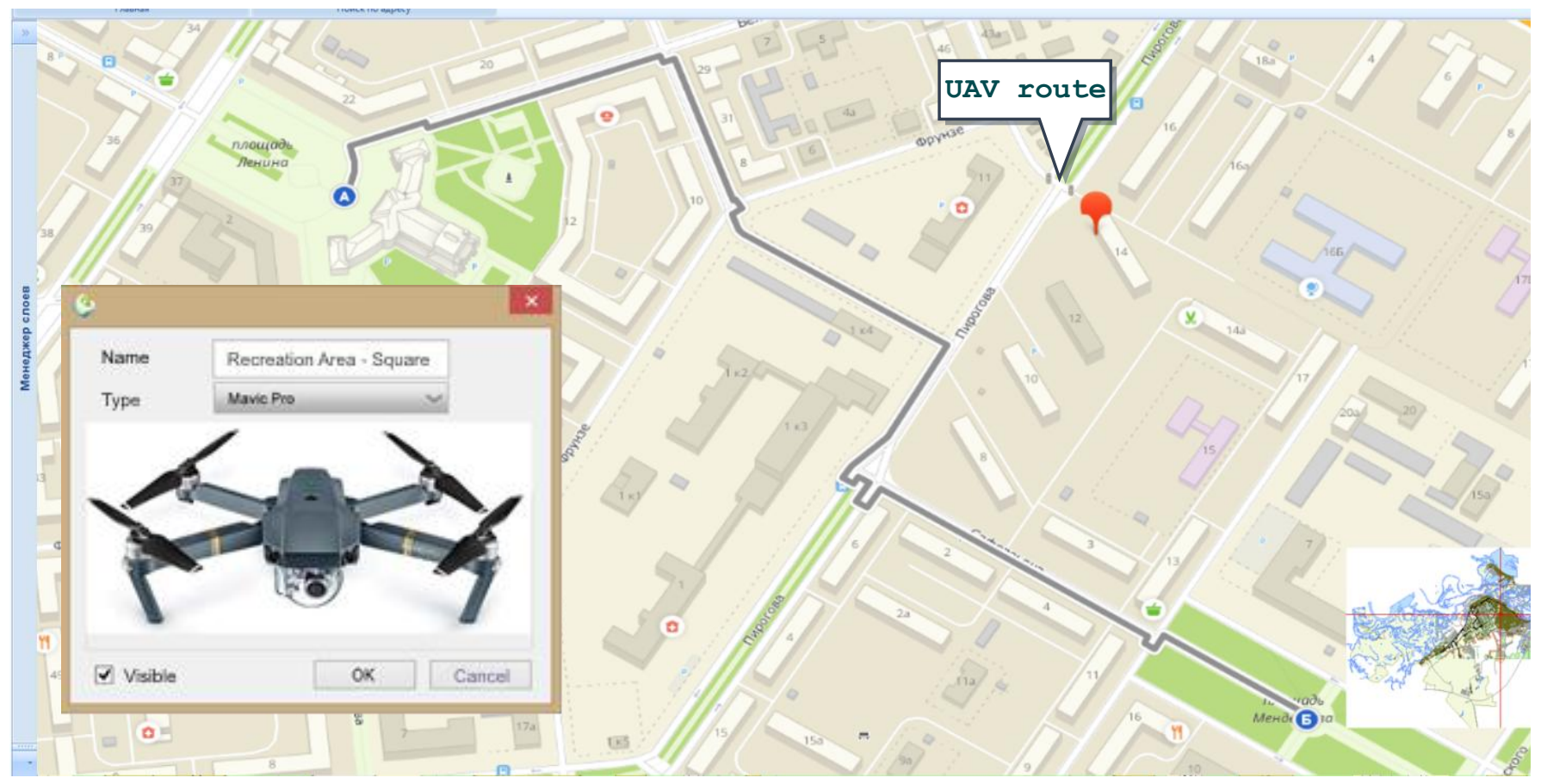

Fig. 3 Construction of the UAV route on the interactive map in the ITSGIS environment 


\section{BUILDING ROUTES ON AN INTERACTIVE MAP}

The described algorithm is used to solve the problem of constructing the trajectory of the territory flight in the environment of intelligent transport geographic information system "ITSGIS", reflecting on the interactive electronic map of the territory of the trajectory (charging stations, landing platforms, etc.), as well as the graph of the transport network. An example of the route is shown in Fig. 3.

The analysis of the algorithm was carried out according to the estimated time of passage of the UAV constructed route at different sizes of the graph of the transport network and different flight conditions. Each cycle automatically generates a graph of the transport network with the specified characteristics [4].

\section{CONCLUSION}

As a result, it is revealed that the advantage of the ant algorithm method is the possibility of finding a rational route, which is achieved by repeated repetition of the procedure of passing agents along the route.

This approach is useful when choosing the shortest route with the use of UAVs in the presence of a significant number of obstacles on the route, perceived as additional reference points.

\section{REFERENCES}

[1] Kozub A. N., Kucherov D.P. Integration approach to the task of choosing the UAV group route // Artificial Intelligence. 2013. №4, S. 333-343.

[2] Alesinskaya T.V. The basics of logistics. General Logistics Management Issues / T.V. Alesinskaya. - Taganrog: Publishing house TRTU, 2005. - $121 \mathrm{~s}$

[3] Mikheeva, T.I. The system for the formation of flight plans for an unmanned aerial vehicle / T.I. Mikheeva, S.V. Mikheev, O.K. Golovnin // Proceedings of the International Technical Conference "Advanced Information Technologies" (PIT-2017). Russia, Samara, Samara University, 2017. S. 669-672.

[4] Mikheeva, T.I. Intelligent transport system. Location of road signs / // Bulletin of Samara State Technical University. Series "Engineering" No. 32. Samara: Samara State Technical University, 2005. S.53-63.
[5] Valeeva A.F., Valeev R.S., Tarasova T.D., Gazizova E.I. On the problem of delivering a homogeneous product to various customers, taking into account the solution of the problems of inventory management, routing and warehousing // Logistics and supply chain management. 2015. №2, S. 54-69.

[6] Pugin, K.V., Efimov S.S. Partial parallelization genetic algorithms in systems with shared memory using the example of the traveling salesman problem // Mathematical Structures and Modeling. 2012. Rel. № 26. S. 110-117.

[7] Shtovba, S.D. Ant algorithms // Exhibitor Pro. Mathematics in Applications. 2013. №4, S. 70-75.

[8] Dorigo M., Gambardella L.M. Ant Colonies for the travelling salesman problem // BioSystems. 1997. № 43. P.73-81

[9] Stutzle T., Hoos H.H. MAX-MIN Ant System // Preprint submitted to Elsiever Science. - 1999.

[10] Bella J. E., McMullen P. R. Ant colony optimization techniques for the vehicle routing problem // Advanced Engineering Informatics. 2004. V. 18. P. 41-48.

[11] Goncharova YU.A. Organization of freight traffic on the basis of an ant colony algorithm based on a population // Actual problems of science and technology: 8th All-Russian Winter School-Seminar for graduate students and young scientists (Ufa, 19-20 february 2013). Ufa: UGATU, 2013. T. 1. S. 109-112.

[12] Valeeva A.F., Goncharova Yu.A. Practical Application of Population Based Ant Colony Optimization Algorithm // ITIDS 2013: Ufa: UGATU. S. 228-231.

[13] Mikheeva T. I. Intelligent transport geoinformation system YTsGIS. Plugins / T.I. Mikheeva, S.V. Mikheev, O.K. i dr. - Samara : Inteltrans, 2016. - T.2. $-217 \mathrm{~s}$.

[14] Karcev N.V., Salykova O.S. UAV flight path planning // Education and science in modern conditions. 2016. №1 (6). S.266-268.

[15] Kulikov G.V., Tambovskij S.S. Assessing the quality of communication with UAVs in urban areas // Russian Technology Journal. 2015. №1(6). S. 205-217.

[16] Mikheeva T.I. Parameterization of control objects of urbanized territories / S.V. Mikheev, O.K. Golovnin // Bulletin of the Samara Scientific Center of the Russian Academy of Sciences. - 2015. - T. 17, № 2 (5). - S. 1058-1062.

[17] Mikheev S.V. Exchange of V2I information in a geographic information transport system in critical situations/ S.V. Mikheev, A.A. Osmushin, O.K. Golovnin // Bulletin of the Samara Scientific Center of the Russian Academy of Sciences. - 2014. - T. 16. - № 4 (2). - S. 399-403. 